\title{
Fisioterapia no controle das emoções durante o pré-natal: Um ensaio clínico
}

\section{randomizado}

\author{
Physiotherapy during prenatal care on the control of emotions: A randomized controlled trial \\ Fisioterapia en el control de las emociones durante la atención prenatal: Un ensayo clínico
}

aleatorizado

Recebido: 05/07/2021 | Revisado: 13/07/2021 | Aceito: 15/07/2021 | Publicado: 25/07/2021

\author{
Marina Baia do Vale \\ ORCID: https://orcid.org/0000-0003-0003-721X \\ Universidade Federal do Pará, Brasil \\ E-mail: marinavalefisioterapeuta@gmail.com \\ Cibele Nazaré Câmara Rodrigues \\ ORCID: https://orcid.org/0000-0002-2429-9970 \\ Universidade Federal do Pará, Brasil \\ E-mail: cibelecamara@hotmail.com \\ Ana Paula Moreira Sales \\ ORCID: https://orcid.org/0000-0002-6839-702X \\ Universidade Federal do Pará, Brasil \\ E-mail: anapaula.apsales@gmail.com \\ Thaynara Luize das Mercês \\ ORCID: https://orcid.org/0000-0003-2016-091X \\ Universidade Federal do Pará, Brasil \\ E-mail: thaymerces94@gmail.com \\ Gissele Maria Figueiredo Nunes \\ ORCID: https://orcid.org/0000-0003-1909-1013 \\ Universidade Federal do Pará, Brasil \\ E-mail: gisse.nunes@gmail.com \\ João Paolo Bilibio \\ ORCID: https://orcid.org/0000-0003-4755-170X \\ Universidade Federal do Pará, Brasil \\ E-mail: joaopaolobilibio@yahoo.com.br
}

\begin{abstract}
Resumo
Objetivo: Analisar a influência da assistência fisioterapêutica pré-natal no controle das emoções. Método: Ensaio clínico randomizado que incluiu primigestas consideradas de risco habitual, cadastradas em pré-natal regular e que desejavam parto normal. As gestantes foram randomizadas em dois grupos, o grupo de fisioterapia, no qual foi aplicado um protocolo de fisioterapia pélvica para preparação do parto normal, e o grupo controle, que não foi submetido ao protocolo de fisioterapia. Em ambos os grupos, ansiedade e depressão foram avaliadas por meio da Escala Hospitalar de Ansiedade e Depressão, e confiança, ansiedade e medo foram avaliadas por meio de um questionário de autopercepção. Resultados: Foram elegíveis 31 gestantes: 16 grupo físioterapia e 15 grupo controle. Foi encontrado um aumento na confiança de $93,3 \%$ versus $30,8 \%$, p 0,003; uma diminuição no medo e ansiedade de $86,7 \%$ versus $13,3 \%, \mathrm{p} 0,031$; e uma influência positiva em relação às técnicas aprendidas que ajudaram ao longo do processo de parto de $86,7 \%$ versus $13,3 \%$, p 0,031 , nos grupos fisioterapia versus controle, respectivamente. Conclusão: O estudo mostrou que a fisioterapia durante a gravidez influencia positivamente a autopercepção do aspecto emocional do parto, aumentando a confiança ( $93 \%$ versus $30 \%)$ e diminuindo a ansiedade e o medo ( $87 \%$ versus $46 \%)$.
\end{abstract}

Palavras-chave: Fisioterapia; Saúde da Mulher; Pré-natal; Emoções; Ansiedade.

\begin{abstract}
Objective: To analyze the influence of prenatal physiotherapy care in the control of emotions. Method: Randomized clinical trial that included primigravidae considered to be of habitual risk, enrolled in regular prenatal care and who wished for vaginal delivery. The pregnant women were randomized into two groups, the physiotherapy group in which a pelvic physiotherapy protocol was applied to prepare for vaginal delivery, and the control group, which was not submitted to the physiotherapy protocol. In both groups anxiety and depression were assessed using the Hospital Anxiety and Depression Scale, and confidence, anxiety and fear were assessed using a self-perception questionnaire. Results: The total of 31 pregnant women were eligible: 16 physical therapy group and 15 control group. There was found an increase in confidence of $93.3 \%$ versus $30.8 \%$, p 0.003 ; a decrease in fear and anxiety of $86.7 \%$ versus
\end{abstract}


$13.3 \%, \mathrm{p} 0.031$; and a positive influence regarding the techniques learned that helped throughout the delivery process of $86.7 \%$ versus $13.3 \%$, p 0.031 , in the physiotherapy versus control groups, respectively. Conclusion: The study showed that physiotherapy during pregnancy positively influences the self-perception of the emotional aspect of childbirth, hence increasing confidence (93\% versus 30\%) and decreasing anxiety and fear (87\% versus $46 \%$ ).

Keywords: Physiotherapy; Women's Health; Prenatal; Emotions; Anxiety.

\section{Resumen}

Objetivo: Analizar la influencia de los cuidados de fisioterapia prenatal en el control de las emociones. Método: Ensayo clínico aleatorizado que incluyó primigrávidas consideradas de riesgo habitual, inscritas en atención prenatal regular y que deseaban parto vaginal. Las gestantes fueron aleatorizadas en dos grupos, el grupo de fisioterapia en el que se aplicó un protocolo de fisioterapia pélvica para preparar el parto vaginal, y el grupo control, que no se sometió al protocolo de fisioterapia. En ambos grupos, la ansiedad y la depresión se evaluaron mediante la Escala Hospitalaria de Ansiedad y Depresión, y la confianza, la ansiedad y el miedo se evaluaron mediante un cuestionario de autopercepción. Resultados: Fueron elegibles 31 gestantes en total: 16 del grupo de fisioterapia y 15 del grupo de control. Se encontró un aumento de la confianza del 93,3\% frente al 30,8\%, p 0,003; una disminución del miedo y la ansiedad del $86,7 \%$ frente al 13,3\%, p 0,031; y una influencia positiva con respecto a las técnicas aprendidas que ayudaron a lo largo del proceso de entrega de $86.7 \%$ versus $13.3 \%$, p 0.031 , en los grupos de fisioterapia versus control, respectivamente. Conclusión: El estudio mostró que la fisioterapia durante el embarazo influye positivamente en la autopercepción del aspecto emocional del parto, aumentando así la confianza (93\% frente al 30\%) y disminuyendo la ansiedad y el miedo ( $87 \%$ frente al $46 \%$ ).

Palabras clave: Fisioterapia; La salud de la mujer; Prenatal; Emociones; Ansiedad.

\section{Introdução}

O parto normal humanizado é proposto e incentivado mundialmente por ser um processo fisiológico que acarreta menos riscos materno-infantis, tendo a parturiente total consciência do que acontece ao seu redor e o poder de escolha durante todo o processo de trabalho de parto (Brasil, 2017). Porém, a primigesta geralmente apresenta medo e ansiedade em relação ao parto normal, sentimentos por vezes relacionados a dor das contrações uterinas e das lacerações perineais espontâneas no momento da expulsão ou da episiotomia (Giacopini; Oliveira \& Araújo, 2016; Smith et al., 2019).

O Brasil apresenta altas taxas de incidência de cesáreas, variando de $40 \%$ nas maternidades públicas a até $86 \%$ nas maternidades privadas (Brasil, 2016). Estes números contrastam com os dos países de primeiro mundo, Estados Unidos 24,7\%, Canadá 19,5\%, Dinamarca 13,1\% e Austrália 7,5\% (Silva et al., 2014). A Organização Mundial de Saúde (OMS) preconiza uma taxa ideal de cesárea entre $10 \%$ e 15\%, ratificando que o procedimento cirúrgico deve ser realizado em casos específicos por indicação médica criteriosa, entretanto, há um crescimento nas taxas de cesárea principalmente em países em desenvolvimento, gerando uma preocupação na comunidade de saúde internacional (Oms, 2018). No Brasil, estudos indicam que existem desigualdades sociais nas taxas de cesárea, sendo essas mais incidentes nos setores privados por mulheres com maior poder aquisitivo e de alta escolaridade, sendo os motivos mais relatados por elas o medo do parto normal por ser uma experiência desconhecida, medo da dor, falta de suporte social, baixa autoestima, e das possíveis lesões de assoalho pélvico adquiridas durante o trabalho de parto (Silva et al., 2014, Smith et al., 2019).

Ainda que o trabalho de parto normal seja o mais recomendado para gestações de risco habitual, este pode acarretar algumas complicações, como as lesões de assoalho pélvico, que no período do pós-parto imediato ou a longo prazo podem causar disfunções dolorosas, sexuais, urinárias e fecais, prejudicando a funcionalidade e qualidade de vida da mulher (Riesco et al., 2011; Elenskaia et al., 2013; Declerq et al., 2014). As lesões de assoalho pélvico apresentam diferentes graus, e dependendo da profundidade da lesão essas podem gerar disfunções permanentes (Frolich \& Ketle, 2015; Leeman 2016; Baracho, 2018).

A fisioterapia na preparação para o parto normal tem como um de seus objetivos atuar na melhora da contração e extensibilidade dos músculos do assoalho pélvico, através de técnicas que promovem o relaxamento e aumentam o alongamento da parede vaginal (Declerq et al., 2013; Baracho, 2018). Dentre as técnicas utilizadas na preparação para o parto 
normal, existem os exercícios pélvicos, a massagem perineal e o uso do perineômetro, que podem gerar resultados positivos como o aumento da confiança da gestante em obter o parto normal, mais rápido, menos doloroso e com menores índices de lacerações espontâneas e episiotomias (Soares et al., 2017; Baracho 2018, Schantz, 2018).

Diante da realidade brasileira de altos índices de cesariana, do medo e ansiedade que as primigestas geralmente sentem em relação ao parto normal, e no contexto da fisioterapia no pré-natal com a finalidade de preparar a musculatura pélvica e assim aumentar a confiança da gestante em relação ao parto normal, o objetivo desse estudo foi analisar a influência da assistência fisioterapêutica pré-natal no controle das emoções.

\section{Metodologia}

\subsection{Tipo de estudo}

Foi realizado um ensaio clínico randomizado em que as participantes foram divididas em dois grupos, o grupo fisioterapia e grupo controle.

\subsection{Local de estudo}

Foi realizada a busca ativa das participantes em seis Unidades Básicas de Saúde e em um Hospital Maternidade. O estudo foi conduzido em um Hospital Maternidade durante o período de setembro de 2018 a setembro de 2019.

\subsection{Aspectos éticos}

O ensaio clínico seguiu com as normas e instruções previstos na Resolução no 466/2012 e 510/2016 do Conselho Nacional de Saúde, foi conduzido de acorda com as normas da CONSORT, aprovado pelo Comitê Nacional de Ética em Pesquisa com número do parecer 2.749.856, registrado e aprovado no Registro Brasileiro de Ensaios Clínicos - REBEC (U1111-1213-8318 - UTN UNIVERSAL TRIAL NUMBER), e todas as participantes assinaram o Termo de Consentimento Livre e Esclarecido como critério para participação do estudo.

\subsection{Critérios de inclusão e exclusão}

Os critérios de inclusão foram primigestas, com idade entre 18 a 35 anos, gestação única, de risco habitual, que estivessem realizando pré-natal regular e que desejassem o parto normal. Gestantes que apresentassem contraindicação para realização de fisioterapia pélvica, que houvessem sofrido cirurgias pélvicas recentes, ou fossem diagnosticadas com depressão ou algum outro distúrbio psicológico foram excluídas do estudo.

\subsection{Cálculo amostral}

Foi baseado no estudo de Toohill e colaboradores (2014) que demonstrou que participantes ( $\mathrm{n}=339)$ de um grupo de intervenção de preparação para o parto $(n=170)$ obtiveram scores significativamente menores em relação ao medo do parto quando comparadas com participantes do grupo controle $(\mathrm{n}=169) ; 47,5 \%$ mulheres do grupo intervenção versus $25,8 \%$ mulheres do grupo controle. O cálculo amostral foi realizado utilizando o software WinPepi, requerendo 31 participantes para esse estudo para um nível de significância de $5 \%$ e poder de $80 \%$.

\subsection{Randomização}

A randomização foi sigilosa e realizada pela randomização em blocos através de web-basedrandomization (Ferreira \& Patino, 2016) e as fichas de avaliação eram catalogadas e organizadas previamente à avaliação inicial. As participantes 
foram randomizadas em dois grupos: Grupo Fisioterapia e Grupo Controle. As participantes de ambos os grupos (Grupo Fisioterapia e Grupo Controle) passaram por três avaliações com a fisioterapeuta: a primeira (avaliação inicial) a partir de 24 semanas de idade gestacional (IG), a segunda (avaliação pré-termo) com 36 semanas de IG e a terceira (avaliação puerperal). Além disso, todas as gestantes receberam orientações, técnicas de posicionamento e respiração para serem realizadas durante o trabalho de parto.

\section{Primeira avaliação (24 semanas de IG)}

Foi realizada a coleta de dados sociodemográficos e clínicos, incluindo a avaliação funcional de assoalho pélvico (AFA) através da palpação digital e escala de Oxford Modificada (Cullerés et al., 2019), e de sintomas de ansiedade e depressão através da Escala Hospitalar de Ansiedade e Depressão (HADS) (Silva et al., 2015).

\section{Segunda avaliação (36 semanas de IG)}

Foi repetido em ambos os grupos o AFA e a escala HADS. Nessa avaliação, também foi entregue às gestantes uma ficha elaborada pelo pesquisador para preenchimento pelo profissional da saúde que iria prestar a assistência ao parto, a qual continha dados referentes ao tipo, tempo e intercorrências do trabalho de parto.

\section{Terceira avaliação (Puerpério imediato - até 10 dias pós-parto)}

A avaliação puerperal foi realizada após alta hospitalar, sendo delimitado até 10 dias pós-parto. As avaliações de puerpério foram realizadas ou no consultório de Fisioterapia no hospital, ou em domicílio, de acordo com a preferência e necessidade das participantes da pesquisa. Para avaliação no puerpério foi utilizada ficha elaborada pelo pesquisador que consistia em dados do parto e do recém-nascido ( $\mathrm{RN}$ ) obtidos pela ficha preenchida pelos profissionais que assistiram ao parto e pela carteira do RN. Nessa avaliação foi utilizado questionário simples com três perguntas acerca da percepção da mulher sobre a fisioterapia na preparação para o parto normal, sendo essas:

1) 'Você sentiu que a preparação para o parto aumentou a sua confiança em obter o parto normal?"'

2) 'Você acha que a preparação para o parto diminuiu o seu medo e ansiedade em relação ao parto?"

3) "Você considera que aprendeu técnicas para utilizar na hora do parto?"

Nessa mesma avaliação também foi aplicada a Escala Visual Analógica (EVA) (Heller, Manuguerra \& Chow, 2016) para avaliar o nível de dor no puerpério e o teste Timed Up and Go (TUG) para avaliar a funcionalidade. Este teste consiste em registrar o tempo que se leva para o participante levantar de uma cadeira, caminhar uma distância de três metros, voltar e se sentar na mesma cadeira (Podisiadlo \& Richardson, 1991). O uso deste teste no contexto do puerpério imediato também foi realizado no estudo de Santos e colaboradores (2016).

As participantes do Grupo Fisioterapia, além das medidas descritas, receberam sessões semanais de fisioterapia especializada na preparação para o parto, agendadas imediatamente após a avaliação inicial. Todas as avaliações e sessões de fisioterapia foram realizadas pela mesma profissional especializada.

\subsection{Protocolo do Grupo Fisioterapia}

O protocolo de fisioterapia foi construído pela equipe de fisioterapia do hospital maternidade no qual a pesquisa foi realizada, e se encontra em processo de validação, sendo baseado primordialmente nos estudos de Alves e colaborares, 2016 e Baracho, 2018. Para o grupo Fisioterapia, após a primeira avaliação e a partir da $24^{\circ}$ semana de IG até a $34^{\circ}$, foi agendado um encontro semanal no ambulatório de Fisioterapia no hospital maternidade, e consistia na realização de seis exercícios para 
fortalecimento do assoalho pélvico em três séries de quinze repetições cada, com um tempo de contração de seis segundos e de relaxamento de três segundos. Os exercícios eram realizados em grupo de forma progressiva e respeitando a especificidade de cada gestante.

Ainda somente para o grupo fisioterapia, a partir da $34^{\circ}$ semana de gestação até a $40^{\circ}$ semana ou data do parto foram interrompidos os exercícios pélvicos e foi realizado técnicas individuais para aumento da extensibilidade e alongamento muscular do assoalho pélvico com a massagem perineal e com o uso do perineômetro vaginal da marca Epi-no® (Alves et al., 2016). A massagem perineal era realizada em dez minutos utilizando a técnica primeiramente descrita por Labrecque e colaboradores (1994), sendo introduzido de um a dois dedos aproximadamente 3 a $4 \mathrm{~cm}$ na vagina, aplicando e mantendo pressão no sentido anteroposterior durante dois minutos e posteriormente nas paredes laterais da vagina por mais dois minutos, sendo repetida a sequência dos movimentos por três vezes utilizando-se lubrificante à base d'água da marca $K Y^{\circledR}$.

A utilização do Epi-no® foi realizada logo após a massagem perineal, posicionando o balão de silicone a aproximadamente $3 \mathrm{~cm}$ no introito vaginal e insuflando o mesmo até a sensação de preenchimento vaginal. A insuflação e desinflação do balão de silicone foi realizada pela própria gestante para maior segurança. Com o dispositivo também foi realizado o treino de expulsão com o balão de silicone insuflado no introito vaginal, objetivando treinar o momento da expulsão do bebê no momento do parto.

\subsection{Análise dos dados}

Os dados foram analisados utilizando o teste $\mathrm{T}$ de Student para variáveis independentes e o teste de Levene para igualdade de variâncias. O teste Qui-Quadrado ou teste exato de Fisher foi utilizado para analisar variáveis categóricas. A razão de possibilidades (oddsratio - OR) e os intervalos de confiança foram calculados. O limiar para significância estatística foi de 5\%. Os testes estatísticos foram realizados utilizando o programa StatisticPackage for the Social Sciences 20 (SPSS Inc., Chicago, USA).

\section{Resultados}

Foram avaliadas para elegibilidade 94 participantes, sendo que 31 foram incluídas no estudo e 63 excluídas, 38 por não comparecerem na avaliação inicial, 23 não se enquadravam aos critérios de inclusão e duas se recusaram a participar da pesquisa (Figura 1). 
Research, Society and Development, v. 10, n. 9, e23810917918, 2021

(CC BY 4.0) | ISSN 2525-3409 | DOI: http://dx.doi.org/10.33448/rsd-v10i9.17918

Figura 1. Diagrama representando o fluxo das participantes em cada etapa da pesquisa.

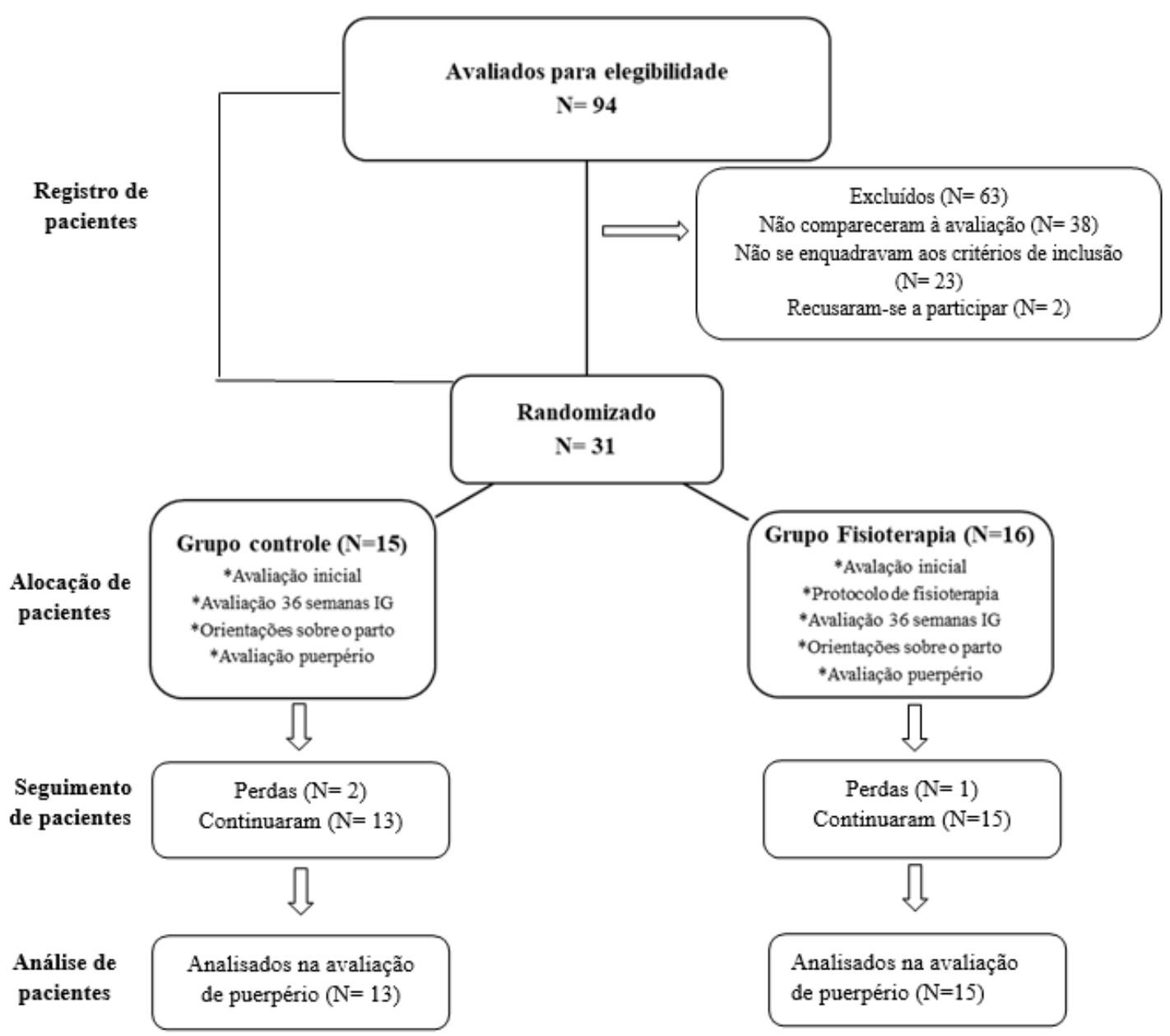

*Intervenções realizadas nos grupos.

Fonte: Autores.

Um total de 31 gestantes foram randomizadas (Grupo Controle $n=15$ e Grupo Fisioterapia n=16). As participantes do Grupo Fisioterapia iniciaram o protocolo fisioterapêutico com uma IG média de $28.8 \pm 3.50$ e realizaram $6.50 \pm 2.68$ sessões, sendo dessas $4.38 \pm 1.92$ de exercícios pélvicos e $2.13 \pm 1.25$ de massagem perineal e uso do Epi-No®. Não houve seguimento de 3 participantes (controle $n=2$, fisioterapia $n=1$ ) após o parto devido perda de contato/falta de retorno delas. Portanto, para análise das fichas de assistência ao parto e avaliação no puerpério foram inclusas 28 participantes.

A análise das características sociodemográficas e clínicas das participantes do grupo controle e fisioterapia

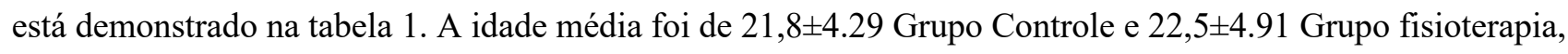
$\mathrm{p}=$ 0.677. A avaliação de força muscular de assoalho pélvico após o protocolo de fisioterapia foi maior no Grupo Fisioterapia 3,77 versus 2,62 no grupo controle, $\mathrm{p}=0.028$. Não encontramos diferença entre os grupos nos demais fatores (idade gestacional, altura, peso, cor, estado civil, escolaridade e renda). 
Research, Society and Development, v. 10, n. 9, e23810917918, 2021

(CC BY 4.0) | ISSN 2525-3409 | DOI: http://dx.doi.org/10.33448/rsd-v10i9.17918

Tabela 1. Análise das características sociodemográficas e clínicas das participantes do grupo controle e fisioterapia (média \pm desvio padrão).

\begin{tabular}{|c|c|c|c|}
\hline Variável & Grupo Controle $(n=15)$ & Grupo Fisioterapia $(n=16)$ & $P$-valor \\
\hline Idade Gestacional (semanas) na $\mathrm{AVi}^{\mathrm{c}}$ & $24.4 \pm 3.15$ & $27.0 \pm 4.09$ & $0.065^{\mathrm{a}}$ \\
\hline Idade (anos) & $21.8 \pm 4.29$ & $22.5 \pm 4.91$ & $0.677^{a}$ \\
\hline Altura $(\mathrm{cm})$ & $1.56 \pm 0.61$ & $1.56 \pm 0.70$ & $0.931^{\mathrm{a}}$ \\
\hline Peso (kg) & $66.4 \pm 11.1$ & $64.0 \pm 10.8$ & $0.559^{\mathrm{a}}$ \\
\hline $\mathrm{AFAi}^{\text {d (grau de força muscular) }}$ & $2.87 \pm 1.64$ & $3.75 \pm 1.06$ & $0.084^{\mathrm{a}}$ \\
\hline $\operatorname{AFAf}^{\prime}$ (grau de força muscular) & $2.62 \pm 1.44$ & $3.77 \pm 1.01$ & $0.028^{\mathrm{a}}$ \\
\hline \multicolumn{4}{|l|}{ Cor da pele } \\
\hline Branca & $0.0 \%$ & $6.3 \%(1)$ & \\
\hline Amarela & $0.0 \%$ & $12.5 \%(2)$ & \\
\hline Parda & $93.3 \%(14)$ & $75.0 \%(12)$ & \\
\hline Negra & $6.7 \%(1)$ & $6.3 \%(1)$ & $0.373^{\mathrm{b}}$ \\
\hline \multicolumn{4}{|l|}{ Estado civil } \\
\hline União estável & $73.3 \%(11)$ & $31.3 \%(5)$ & \\
\hline Casada & $6.7 \%(1)$ & $18.8 \%(3)$ & \\
\hline Solteira & $20.0 \%(3)$ & $50.0 \%(8)$ & $0.064^{\mathrm{b}}$ \\
\hline \multicolumn{4}{|l|}{ Escolaridade } \\
\hline Fundamental & $40.0 \%(6)$ & $12.5 \%(2)$ & \\
\hline Médio & $46.7 \%(7)$ & $62.6 \%(10)$ & \\
\hline Superior & $13.4 \%(2)$ & $25.1 \%(4)$ & $0.568^{b}$ \\
\hline \multicolumn{4}{|l|}{ Renda familiar } \\
\hline$<1$ salário & $73.3 \%(11)$ & $68.8 \%(11)$ & \\
\hline 1 salário & $20.0 \%(3)$ & $18.8 \%(3)$ & \\
\hline$>1$ salário & $6.7 \%(1)$ & $12.5 \%(2)$ & $0.396^{\mathrm{b}}$ \\
\hline \multicolumn{4}{|l|}{ Profissão } \\
\hline Do lar & $26.0 \%(4)$ & $43.8 \%(7)$ & \\
\hline Estudante & $33.3 \%(5)$ & $18.8 \%(3)$ & \\
\hline Lavradora & $6.7 \%(1)$ & $0.0 \%$ & \\
\hline Secretária & $0.0 \%$ & $12.5 \%(1)$ & \\
\hline Outros & $33.3 \%(5)$ & $25.0 \%(4)$ & $0.354^{\mathrm{b}}$ \\
\hline
\end{tabular}

${ }^{a}$ Teste T de Student.

b Teste Qui-Quadrado.

${ }^{c}$ Avaliação inicial.

d Avaliação Funcional do Assoalho Pélvico Inicial.

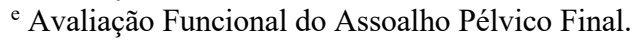

Fonte: Autores.

A análise da Ansiedade e Depressão pré e pós protocolo de fisioterapia de acordo com a Escala Hospitalar de Ansiedade e Depressão está demonstrada na tabela 2. Não encontramos diferença na avaliação da ansiedade e depressão pré e pós fisioterapia entre os grupos. 
Research, Society and Development, v. 10, n. 9, e23810917918, 2021

(CC BY 4.0) | ISSN 2525-3409 | DOI: http://dx.doi.org/10.33448/rsd-v10i9.17918

Tabela 2. Ansiedade e depressão pré e pós protocolo de Fisioterapia de acordo com a tabela HADS*.

\begin{tabular}{|c|c|c|c|c|c|c|c|c|}
\hline \multirow[t]{2}{*}{ Variáveis } & \multicolumn{2}{|c|}{ Grupo Controle $(\mathrm{n}=15)$} & \multirow[t]{2}{*}{ OR $(95 \%)$} & \multirow[t]{2}{*}{ P-valor ${ }^{\mathbf{a}}$} & \multicolumn{2}{|c|}{ Grupo Fisioterapia $(n=16)$} & \multirow[t]{2}{*}{$\begin{array}{c}\text { OR } \\
(95 \%)\end{array}$} & \multirow[t]{2}{*}{ P-valor } \\
\hline & Pré Protocolo & Pós Prot & & & Pré Protocolo & Pós Protocolo & & \\
\hline $\begin{array}{r}\text { Ansiedade } \\
\%,(n)\end{array}$ & $53.3 \%(8)$ & $33.3 \%(5)$ & $0.4(0.1-1.9)$ & $0.272^{b}$ & $31.2 \%(5)$ & $31.2 \%(5)$ & $\begin{array}{r}1.0 \\
(0.24 .4)\end{array}$ & 1.000 \\
\hline $\begin{array}{l}\text { Ansiedade } \\
\text { média } \pm \text { DP }\end{array}$ & $7.2 \pm 4.1$ & $7.7 \pm 3.6$ & - & 0.618 & $7.9(4.1)$ & $8.2(2.3)$ & - & 0.770 \\
\hline $\begin{array}{r}\text { Depressão \%, } \\
(\mathbf{n})^{\mathrm{c}}\end{array}$ & $25.0 \%(3)$ & $16.7 \%(2)$ & $1.6(0.2-11.5)$ & 0.626 & $35.7 \%(5)$ & $21.4 \%(3)$ & $\begin{array}{r}0.4 \\
(0.092 .6)\end{array}$ & 0.406 \\
\hline $\begin{array}{r}\text { Depressão, } \\
\text { Média }^{c}\end{array}$ & $4.6(3.5)$ & $5.2(3.6)$ & - & 0.589 & $6.6(3.2)$ & $6.8(3.1)$ & - & 0.702 \\
\hline
\end{tabular}

*Escala Hospitalar de Ansiedade e Depressão

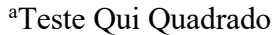

${ }^{\mathrm{b}}$ Teste T Pareado

${ }^{\mathrm{c}}$ Nessa análise somente 12 participantes do grupo controle e 14 do grupo fisioterapia foram incluídas devido a perda amostral.

DP: Desvio Padrão

Fonte: Autores.

A avaliação das características do parto das participantes do grupo controle e fisioterapia estão demonstradas na tabela 3. Apenas oito pacientes evoluíram para parto normal em todos os grupos (grupo controle 38.5\% versus 20.0\% grupo fisioterapia, p 0.613), sendo que o principal motivo da indicação de cesáreas foi o sofrimento fetal (37.5\% controle versus $50 \%$ fisioterapia, p 0.531). A IG média de nascimento entre os grupos foi semelhante (39.31\% controle versus $39.13 \%$ fisioterapia, p 0.798). A posição durante o período expulsivo das gestantes que tiveram parto normal foi significativamente maior em decúbito dorsal na cama ginecológica no grupo controle $62.5 \%$ versus $12.5 \%$ grupo fisioterapia, p 0.035 . A apresentação pélvica foi mais prevalente no grupo fisioterapia $26.7 \%$ versus $0 \%$ grupo controle, p 0.044 . 
Research, Society and Development, v. 10, n. 9, e23810917918, 2021

(CC BY 4.0) | ISSN 2525-3409 | DOI: http://dx.doi.org/10.33448/rsd-v10i9.17918

Tabela 3. Características do parto das participantes do grupo controle e fisioterapia.

\begin{tabular}{|c|c|c|c|}
\hline Variáveis & Grupo controle $(n=13)$ & Grupo fisioterapia (n=15) & $P$-valor \\
\hline Parto normal \% (n) & $38.5 \%(5)$ & $20.0 \%(3)$ & \\
\hline Parto cesárea \% (n) & $61.5 \%(8)$ & $80.0 \%(12)$ & $0.613^{\mathrm{a}}$ \\
\hline Idade Gestacional no parto (semanas) & $39.31 \pm 1.70$ & $39.13 \pm 1.84$ & $0.798^{\mathrm{a}}$ \\
\hline Tempo de trabalho de parto (minutos) & $514.6 \pm 526.1$ & $3336.5 \pm 313.8$ & $0.246^{\mathrm{C}}$ \\
\hline \multicolumn{4}{|l|}{ Posição durante trabalho de parto } \\
\hline Decúbito dorsal no leito & $0.0 \%$ & $25.0 \%(\mathrm{n}=2)$ & \\
\hline Decúbito dorsal na cama ginecológica & $62.5 \%(\mathrm{n}=5)$ & $12.5 \%(\mathrm{n}=1)$ & $0.035^{b}$ \\
\hline \multicolumn{4}{|l|}{ Apresentação fetal (última USG) } \\
\hline Cefálica & $46.4 \%(n=13)$ & $73.3 \%(\mathrm{n}=11)$ & \\
\hline Pélvica & $0.0 \%$ & $26.7 \%(\mathrm{n}=4)$ & $0.044^{b}$ \\
\hline Episiotomia/Episiorrafia & $20 \%(n=1)$ & $0.0 \%$ & $0.408^{b}$ \\
\hline \multicolumn{4}{|l|}{ Laceração (grau) } \\
\hline 0 & $20.0 \%(\mathrm{n}=1)$ & $66.7 \%(\mathrm{n}=2)$ & \\
\hline 1 & $60.0 \%(\mathrm{n}=3)$ & $33.3 \%(\mathrm{n}=1)$ & \\
\hline 2 & $20.0 \%(\mathrm{n}=1)$ & $0.0 \%$ & $0.376^{\mathrm{b}}$ \\
\hline \multicolumn{4}{|l|}{ Motivo de cesárea } \\
\hline Falha na indução & $12.5 \%(\mathrm{n}=1)$ & $16.7 \%(\mathrm{n}=2)$ & \\
\hline Sofrimento fetal & $37.5 \%(\mathrm{n}=3)$ & $50.0 \%(\mathrm{n}=6)$ & \\
\hline Doença hipertensiva da gravidez & $37.5 \%(\mathrm{n}=3)$ & $8.3 \%(\mathrm{n}=1)$ & \\
\hline Desproporção céfalo-pélvica & $12.5 \%(\mathrm{n}=1)$ & $25.0 \%(n=3)$ & $0.531^{b}$ \\
\hline
\end{tabular}

${ }^{\mathrm{a}}$ Teste T Student.

b Teste Exato de Fisher/Qui-Quadrado.

${ }^{\mathrm{c}}$ Teste de Mann-Whitney.

Fonte: Autores.

A avaliação da dor e funcionalidade no pós-parto nos grupos controle e fisioterapia através da Escala Visual Analógica de Dor (EVA) e teste Timed Up and Go (TUG) estão demonstrados na tabela 4. A média da avaliação em dias pósparto foi de $19.3 \pm 9.96$ para o grupo controle e $23.6 \pm 15.9$ para o grupo fisioterapia, p 0,43 . Não encontramos diferença na dor pós-parto e na funcionalidade entre os grupos.

Tabela 4. Dor e funcionalidade no pós-parto nos grupos controle e fisioterapia através da Escala Visual Analógica de Dor (EVA) e teste Timed Up and Go (TUG) (média \pm desvio padrão).

\begin{tabular}{lccc}
\hline \multicolumn{1}{c}{ Variáveis } & Grupo controle (n= 13) & Grupo fisioterapia (n=15) & $P$-valor ${ }^{\mathbf{a}}$ \\
\hline Dor pós-parto (Escala EVA numérica) & $2.85 \pm 3.23$ & $3.80 \pm 2.78$ & 0.409 \\
Funcionalidade (TUG em segundos) & $12.77 \pm 5.49$ & $13.60 \pm 6.93$ & 0.731 \\
\hline
\end{tabular}

Teste T Student.

Fonte: Autores.

A análise da percepção das participantes em relação ao protocolo de preparação para o parto está demonstrada na tabela 5. Ao serem questionadas sobre a percepção delas acerca do protocolo fisioterapêutico, a maioria do grupo fisioterapia relatou que a preparação para o parto teve influência no aumento da sua confiança (93.3\% versus 30.8\%, p 0,003) com um OR de 31,5 (IC 3,0 - 328,9 P 0,003), diminuição do medo e ansiedade (86.7\% versus 13.3\%, p 0,031) com OR de 7,6 (IC 1,2 - 
Research, Society and Development, v. 10, n. 9, e23810917918, 2021

(CC BY 4.0) | ISSN 2525-3409 | DOI: http://dx.doi.org/10.33448/rsd-v10i9.17918

48,0, P 0,031), e influenciado positivamente no aprendizado de técnicas que as auxiliaram durante todo o processo do trabalho de parto (86.7\% versus 13.3\%, P 0.031, ) com OR de 7,5 (IC 1,2 - 41,0 P 0,031).

Tabela 5. Análise da percepção das participantes em relação ao protocolo de preparação para o parto.

\begin{tabular}{|c|c|c|c|c|}
\hline Variável & $\begin{array}{l}\text { Grupo controle } \\
(n=13)\end{array}$ & $\begin{array}{l}\text { Grupo fisioterapia } \\
(n=15)\end{array}$ & OR (IC 95\%) & $P$-valor ${ }^{a}$ \\
\hline \multicolumn{5}{|l|}{ Aumento da confiança } \\
\hline Muito & $30.8 \%(n=4)$ & $93.3 \%(n=14)$ & & \\
\hline Um pouco & $53.8 \%(\mathrm{n}=7)$ & $6.7 \%(n=1)$ & & \\
\hline Não fez diferença & $15.4 \%(n=2)$ & $0.0 \%(n=0)$ & $31,5(3,0-328,9)$ & 0.003 \\
\hline \multicolumn{5}{|l|}{ Diminuição da ansiedade e medo } \\
\hline Muito & $46.2 \%(\mathrm{n}=6)$ & $86.7 \%(n=13)$ & & \\
\hline Um pouco & $38.5 \%(\mathrm{n}=5)$ & $13.3 \%(\mathrm{n}=2)$ & & \\
\hline Não fez diferença & $15.4 \%(\mathrm{n}=2)$ & $0.0 \%(\mathrm{n}=0)$ & $7,6(1,2-48,0)$ & 0.031 \\
\hline \multicolumn{5}{|l|}{ Aprendizado de técnicas } \\
\hline Muito & $46.2 \%(n=6)$ & $86.7 \%(n=13)$ & & \\
\hline Um pouco & $46.2 \%(\mathrm{n}=6)$ & $13.3 \%(\mathrm{n}=2)$ & & \\
\hline Não fez diferença & $7.7 \%(\mathrm{n}=1)$ & $0.0 \%(\mathrm{n}=0)$ & $7,5(1,2-41,0)$ & 0.031 \\
\hline
\end{tabular}

${ }^{\text {a }}$ Teste Exato de Fisher/Qui-Quadrado.

Fonte: Autores.

\section{Discussão}

A gestação e o período do parto e puerpério causam um grande aumento da ansiedade e do medo para as gestantes (Huizink, 2016; Silva, 2017; Koc et al., 2020). Este estudo encontrou que a fisioterapia no período gestacional tem influência positiva no aspecto emocional da gestante que realiza a preparação para o parto. Houve um aumento da confiança nas pacientes submetidas a fisioterapia de $93 \%$ versus $30 \%$ no grupo controle, enquanto $86 \%$ versus $46 \%$ relataram diminuição do medo e ansiedade, e ainda o aprendizado de técnicas que as auxiliaram durante o parto (de posicionamento e respiração).

Apesar da maioria das pacientes do nosso estudo ter evoluído para a cesárea, elas se sentiram mais seguras e confiantes durante o período do pré-natal, sentimentos esses importantes para as primigestas, pois os efeitos de saúde adversos materno-infantis podem ser advindos das preocupações e ansiedade durante a gestação (Silva, 2017; Huizink, 2016).

Em relação aos programas de preparação para o parto, a maioria dos estudos relacionados a fisioterapia no pré-natal têm como objetivo principal analisar os desfechos de lesão perineal e não o impacto nas emoções (Pereira, et al., 2015; Alves, et al. 2016). Porém, uma revisão sistemática acerca da eficácia do Epi-No® no pré-natal corrobora com os achados do nosso estudo, pois afirma que gestantes que se preparam para o parto com o equipamento se sentem mais confiantes, e aprendem técnicas de controle de dor e de estímulos para o trabalho de parto (Pereira et al., 2015).

No estudo de Stoll e colaboradores (2018), foram analisadas as intervenções não farmacológicas durante um programa de pré-natal para diminuição da ansiedade e medo do parto, sendo destacadas como efetivos os exercícios físicos, a prática de Yoga e os cursos de educação no pré-natal. Outro ensaio clínico randomizado, avaliou a influência de um programa de preparação para o parto nos desfechos materno-infantis. O estudo não comprovou a influência do programa na diminuição da ansiedade, porém apresentou efeito positivo no encorajamento a realização de exercícios físicos durante a gestação. O 
programa também não demonstrou efeitos adversos na gestação, corroborando com os achados do nosso estudo (Miquelutti, Cecatti \& Makuchi, 2013).

No estudo prospectivo randomizado controlado de Çankaya e Simsek (2021), foi demonstrado que um programa de educação pré-natal teve impacto positivo no controle das emoções de primigestas. O grupo intervenção apresentou menor índice de medo, ansiedade e depressão no pós-parto quando comparado aos grupos que não receberam as orientações.

Neste estudo, um total de $41,9 \%$ das participantes apresentou ansiedade e depressão de acordo com a escala HADS, corroborando com os estudos de Silva e colaboradores (2015) acerca dos sintomas psicológicos em primigestas, onde a ansiedade se encontra mais prevalente devido ao medo e a expectativa nessa fase. Alguns fatores de risco para desenvolvimento de ansiedade e depressão durante a gestação são o desemprego, pouco suporte social e primeira gestação, sendo que os fatores benéficos são os exercícios físicos, o suporte familiar, social e intervenções durante o início da gestação (Xian et al., 2019). Apesar do presente estudo não ter demonstrado diferença nos sintomas de ansiedade e depressão antes e após o protocolo de fisioterapia pela escala HADS, foram obtidos excelentes resultados a partir da percepção das participantes da pesquisa, ratificando assim a importância de estratégias que promovam saúde global materno-infantil durante o pré-natal, priorizando também os aspectos emocionais.

Em relação ao tipo de parto das participantes da pesquisa, tanto o grupo controle quanto o grupo fisioterapia apresentaram altas taxas de cesarianas, fato este que condiz com a alta incidência de cesáreas em primigestas no Brasil (Silva et al., 2015; Brasil 2016). Nesta pesquisa, a indicação das cesáreas enquadrou-se com as recomendações do Ministério da Saúde, pois, a maioria das gestantes apresentou sofrimento fetal, desproporção céfalo-pélvica e doenças hipertensivas da gestação (Brasil, 2016). Apesar de todas as participantes do estudo realizarem pré-natal de forma regular e não apresentarem alto risco na avaliação inicial, algumas apresentaram complicações ao final da gestação e no momento do parto, sendo esses indicativos de cesárea de urgência.

Outro ponto importante a ser observado acerca do tipo de parto é que os hospitais públicos brasileiros ainda estão se adequando às recomendações que a Organização Mundial de Saúde lançou no ano de 2018 sobre a assistência durante o parto. Dentre as 56 recomendações, destacam-se a comunicação efetiva entre os profissionais de saúde e familiares, métodos não farmacológicos para alívio da dor durante o trabalho de parto, intervenção médica na dilatação do colo uterino conforme evolução do partograma e analgesia no parto (Oms, 2018).

No contexto dos métodos para alívio da dor durante o trabalho de parto, todos os profissionais da saúde podem prestar assistência à parturiente, e o fisioterapeuta que atua em sala de parto é capacitado para a realização dos procedimentos assegurados pelo Ministério da Saúde (Brasil, 2017; Baracho, 2018). No estudo de Balieiro e colaboradores (2021) foi demonstrado que gestantes que receberam a assistência fisioterapêutica durante o trabalho de parto tiveram uma experiência positiva, pois se sentiram como um membro útil e cooperativo com a equipe.

Encontramos diferença entre os grupos no posicionamento durante o trabalho de parto (TP), no qual as participantes do grupo fisioterapia optaram mais pela posição em decúbito dorsal (DD) no leito, enquanto as participantes do grupo controle em DD na cama ginecológica. Esse resultado pode ter sido em decorrência das orientações sobre o posicionamento adequado durante o TP, pois a cama ginecológica não é a mais apropriada para a passagem do bebê, por não permitir a posição de nutação pélvica e maior alargamento pélvico (Brasil, 2017; Baracho, 2018). A apresentação pélvica também foi mais prevalente no grupo fisioterapia, o que levou ao resultado de mais indicações de cesáreas por apresentação fetal anormal no grupo. O resultado diferenciado entre os grupos não apresenta relação com as técnicas fisioterapêuticas realizadas durante a preparação para o parto, pois não foi realizada nenhuma abordagem para mudança de posicionamento fetal. 
$\mathrm{Na}$ avaliação de puerpério, não encontramos diferença na dor e funcionalidade das participantes na EVA e no teste TUG. Os estudos mostram que a via de parto cesáreo traz maior limitação funcional, dor e insatisfação no pós-parto (Domingues, 2014; Santos 2016), porém não houve a possibilidade de realizar a comparação acerca da via de parto devido à baixa prevalência de partos normais. Ademais, a dificuldade de seguimento no tempo adequado das participantes da pesquisa no pós-parto imediato também foi um fator de influência nos resultados acerca da funcionalidade, pois, apesar de termos delimitado até 10 dias pós-parto para a avaliação de puerpério, a maioria das pacientes não teve a possibilidade de comparecer a consulta fisioterapêutica na data agendada, tendo que ser remarcado ou realizada a avaliação em domicílio após o puerpério imediato.

Em relação ao perfil sociodemográfico das participantes, este é compatível com os achados de estudos epidemiológicos de gestantes usuárias de serviços públicos de saúde (Gomes, 2013; Moussa, Santos \& Almeida, 2014; Brasil, 2014). A renda familiar menor que um salário-mínimo, a cor da pele parda e o estado civil estão de acordo com os achados da região Norte no censo Nacional de 2014 (Brasil, 2014). Na avaliação inicial de grau de força muscular do assoalho pélvico, a maioria apresentava grau de força 2 e 3, considerado pela literatura um grau de força muscular baixo a moderado (Cullerés et al. 2019). Porém, após aplicação do protocolo de Fisioterapia (com 36 semanas de IG) o grupo fisioterapia apresentou ganho de força muscular em assoalho pélvico, corroborando com a literatura (Baracho 2018, Jacomo, et al., 2020).

Este estudo cumpriu o objetivo de analisar a influência da assistência fisioterapêutica pré-natal no controle das emoções demonstrando que o protocolo fisioterapêutico de preparação para o parto corroborou para a diminuição do medo e ansiedade em relação ao parto. Como limitação, obtivemos perda amostral no seguimento de participantes no período puerperal, além de dificuldade na realização da avaliação puerperal com até 10 dias pós-parto, o que influenciou na análise da dor e da funcionalidade no pós-parto imediato. Sugerimos para futuros estudos a delimitação dessa avaliação preferencialmente em domicílio, pois as puérperas se encontram com dificuldade de locomoção e disposição para comparecer ao consultório nesse período.

Para a realização de estudos futuros sobre a influência da fisioterapia nos desfechos neonatais será necessária uma amostra maior para termos um poder adequado e serem obtidas as comparações necessárias. É imprescindível também a busca por incentivos educacionais como palestras e rodas de conversa com as gestantes no pré-natal, para oferecê-las conhecimento acerca do tema e incentivá-las na maior participação e adesão ao protocolo fisioterapêutico.

\section{Conclusão}

O estudo mostrou eficácia do protocolo fisioterapêutico de preparação para o parto normal em primigestas no aumento da confiança e diminuição da ansiedade e do medo, além do aumento do aprendizado de técnicas que lhes auxiliaram durante todo o processo de trabalho de parto. Portanto, a preparação da fisioterapia durante o período gestacional influencia positivamente no controle das emoções. Faz-se necessário estudos futuros com uma amostra adequada para avaliar o impacto da fisioterapia nas características neonatais, do parto e pós-parto.

\section{Agradecimentos}

À todas as gestantes que participaram desse estudo e às equipes multiprofissionais do Hospital Santo Antônio Maria Zaccaria e das Unidades Básicas de Saúde do município de Bragança/PA. 
Research, Society and Development, v. 10, n. 9, e23810917918, 2021

(CC BY 4.0) | ISSN 2525-3409 | DOI: http://dx.doi.org/10.33448/rsd-v10i9.17918

\section{Referências}

Alves, P., Cavalli, R., Bertacini D., \& Driusso, P. (2016). Effects of antenatal pelvic floor preparation techniques on the perineal integrity: a randomized controlled trial protocol. International Journal of Clinical Trials. 3 (4). http://dx.doi.org/10.18203/2349-3259.ijct20163958

Balieiro, J., Silva, A., Mendonça T., Rodrigues, L., Melo-Neto J., Vale M., \& Rodrigues C. (2021). Efeitos da fisioterapia durante o parto em um hospital da região amazônica: Um estudo piloto. Research, Society and Development. 10 (6). http://dx.doi.org/10.33448/rsd-v10i6.16115

Baracho E. (2018). Fisioterapia aplicada à saúde da mulher. Guanabara Koogan

Brasil. (2014). Uma análise das condições de vida da população brasileira. Síntese de indicadores sociais. Instituto Brasileiro de Geografia e Estatística

Brasil. (2016). Sistema de Informações sobre Nascidos Vivos. Ministério da Saúde. DataSus. Brasília-DF.

http://www2.datasus.gov.br/DATASUS/index.php?area=060702

Brasil. (2017). Diretrizes nacionais de assistência ao parto normal. Ministério da Saúde. Secretaria de Ciência, Tecnologia e Insumos Estratégicos. Departamento de Gestão e Incorporação de Tecnologias em Saúde. Brasília-DF.

https://bvsms.saude.gov.br/bvs/publicacoes/diretrizes_nacionais_assistencia_parto_normal.pdf

Çankaya, S., \& Şimşek, B. (2021). Effects of Antenatal Education on Fear of Birth, Depression, Anxiety, Childbirth Self-Efficacy, and Mode of Delivery in Primiparous Pregnant Women: A Prospective Randomized Controlled Study. Clinical nursing research, 30(6), 818-829.

https://doi.org/10.1177/1054773820916984

Declercq, E. R., Sakala, C., Corry, M. P., Applebaum, S., \& Herrlich, A. (2014). Major Survey Findings of Listening to Mothers (SM) III: New Mothers Speak Out: Report of National Surveys of Women's Childbearing ExperiencesConducted October-December 2012 and January-April 2013. The Journal of perinatal education, 23(1), 17-24. https://doi.org/10.1891/1058-1243.23.1.17

Domingues, R., Dias, M., Pereira, M., Torres J., d'Orsi, E., Pereira, A., Schilithz, A., \& Leal, M. (2014) Processo de decisão pelo tipo de parto no Brasil: da preferência inicial das mulheres à via de parto final. Cad.Saúde Pública. 30 S101-S116

Elenskaia, K., Thakar, R., Sultan, A. H., Scheer, I., \& Onwude, J. (2013). Effect of childbirth on pelvic organ support and quality of life: a longitudinal cohort study. International urogynecology journal, 24(6), 927-937. https://doi.org/10.1007/s00192-012-1932-7

Ferreira, J., \& Patino, C. (2016) Randomization: beyond tossing a coin. J Bras Pneumol. 42(5):310-310. https://doi.org/10.1590/S1806-37562016000000296

Giacopini, S., Oliveira, D., \& Araújo, A. (2016). Benefícios e Recomendações da Prática de Exercícios Físicos na Gestação. Revista BioSalus.1:1-19

Gomes, R. M. T., \& César, J. A. (2013). Perfil epidemiológico de gestantes e qualidade do pré-natal em unidade básica de saúde em Porto Alegre, Rio Grande do Sul, Brasil. Revista Brasileira De Medicina De Família E Comunidade, 8(27), 80-89. https://doi.org/10.5712/rbmfc8(27)241

Heller, G. Z., Manuguerra, M., \& Chow, R. (2016). How to analyze the Visual Analogue Scale: Myths, truths and clinical relevance. Scandinavian journal of pain, 13, 67-75. https://doi.org/10.1016/j.sjpain.2016.06.012

Jacomo, R. H., Nascimento, T. R., Lucena da Siva, M., Salata, M. C., Alves, A. T., da Cruz, P., \& Batista de Sousa, J. (2020). Exercise regimens other than pelvic floor muscle training cannot increase pelvic muscle strength-a systematic review. Journal of bodywork and movement therapies, 24(4), 568-574. https://doi.org/10.1016/j.jbmt.2020.08.005

Koc, A. E., Colak, S., Colak, G. V., Pusuroglu, M., \& Hocaoglu, C. (2021). Investigating fear of childbirth in pregnant women and its relationship between anxiety sensitivity and somatosensory amplification. Journal of obstetrics and gynaecology : the journal of the Institute of Obstetrics and Gynaecology, 41(2), 217-223. https://doi.org/10.1080/01443615.2020.1732894

Labrecque, M., Marcoux, S., Pinault, J. J., Laroche, C., \& Martin, S. (1994). Prevention of perineal trauma by perineal massage during pregnancy: a pilot study. Birth (Berkeley, Calif.), 21(1), 20-25. https://doi.org/10.1111/j.1523-536x.1994.tb00911.x

Leeman, L., Rogers, R., Borders, N., Teaf, D., \& Qualls, C. (2016). The Effect of Perineal Lacerations on Pelvic Floor Function and Anatomy at 6 Months Postpartum in a Prospective Cohort of Nulliparous Women. Birth (Berkeley, Calif.), 43(4), 293-302. https://doi.org/10.1111/birt.12258

Miquelutti, M. A., Cecatti, J. G., \& Makuch, M. Y. (2013). Evaluation of a birth preparation program on lumbopelvic pain, urinary incontinence, anxiety and exercise: a randomized controlled trial. BMC pregnancy and childbirth, 13, 154. https://doi.org/10.1186/1471-2393-13-154

Moussa, L., Santos, C., \& Almeida, M. (2014) Percepção da qualidade de vida e prevalência de sintomas urinários em primigestas. Linkania Revista.v8(1):6.

Pereira, A., Oliveira, A., Cury, G., Jorge, L., \& Latorre, G. (2015). Eficácia do Epi-No® na diminuição da episiotomia e risco de lesão perineal pós-parto: revisão sistemática. FEMINA. 43 (6). http://files.bvs.br/upload/S/0100-7254/2015/v43n6/a5324.pdf

Podsiadlo, D., \& Richardson, S. (1991). The timed "Up \& Go": a test of basic functional mobility for frail elderly persons. Journal of the American Geriatrics Society, 39(2), 142-148. https://doi.org/10.1111/j.1532-5415.1991.tb01616.x

Riesco, M., Costa, A., Almeida, S., Basile, A., \& Oliveira, S. (2011). Episiotomia, laceração e integridade perineal em partos normais: análise de fatores associados. Rev. enferm. UERJ. 19(1):77-83. https://pesquisa.bvsalud.org/portal/resource/pt/lil-591019

Romero-Cullerés, G., Jané-Feixas, C., Vilaseca-Grané, A., Arnau, A., Montesinos, J., \& Abenoza-Guardiola, M. (2019). Inter-rater reliability of the digital palpation of pelvic floor muscle by the modified Oxford Grading Scale in continent and incontinent women. Fiabilidad inter evaluador de la palpación digital 
Research, Society and Development, v. 10, n. 9, e23810917918, 2021

(CC BY 4.0) | ISSN 2525-3409 | DOI: http://dx.doi.org/10.33448/rsd-v10i9.17918

de los músculos del suelo pélvico utilizando la escala modificada de Oxford graduada en mujeres continentes e incontinentes. Archivos espanoles de urologia, 72(6), 602-607

Santos, P., Rett, M., Lotti, R., Moccellin, A., \& DeSantana, J. (2016) A vida de parto interfere nas atividades cotidianas no puerpério imediato? ConScientiae Saúde. 15(4):604-611. https://pesquisa.bvsalud.org/portal/resource/pt/biblio-846735

Silva, S., Prates, R., \& Campelo, B. (2014). Parto normal ou cesariana? Fatores que influenciam na escolha da gestante. Revista de Enfermagem da UFSM, 4(1), 1 - 9. doi:https://doi.org/10.5902/217976928861

Silva, M., Leite, E., Nogueira, D., \& Clapis, M. (2017). Anxiety in pregnancy: prevalence and associated factors. Rev enferm UFPE online. 9(7): 9027-37. https://doi.org/10.1590/S1980-220X2016048003253

Soares, D., Soares, J., Graup, S., \& Streb, A. (2017). Atividade física na gestação: uma revisão integrativa. Rev Perspect. Ci. e Saúde. 2(2): 71-84. http://sys.facos.edu.br/ojs/index.php/perspectiva/article/view/138

Schantz C. (2018). Quelles interventions au cours de la grossesse diminuent le risque de lésions périnéales ? RPC Prévention et protection périnéale en obstétrique CNGOF [Methods of preventing perineal injury and dysfunction during pregnancy: CNGOF Perineal prevention and protection in obstetrics]. Gynecologie, obstetrique, fertilite \& senologie, 46(12), 922-927. https://doi.org/10.1016/j.gofs.2018.10.027

Smith, L. A., Price, N., Simonite, V., \& Burns, E. E. (2013). Incidence of and risk factors for perineal trauma: a prospective observational study. BMC pregnancy and childbirth, 13, 59. https://doi.org/10.1186/1471-2393-13-59

Smith, V., Gallagher, L., Carroll, M., Hannon, K., \& Begley, C. (2019). Antenatal and intrapartum interventions for reducing caesarean section, promoting vaginal birth, and reducing fear of childbirth: An overview of systematic reviews. PloS one, 14(10), e0224313. https://doi.org/10.1371/journal.pone.0224313

Stoll, K., Swift, E. M., Fairbrother, N., Nethery, E., \& Janssen, P. (2018). A systematic review of nonpharmacological prenatal interventions for pregnancyspecific anxiety and fear of childbirth. Birth (Berkeley, Calif.), 45(1), 7-18. https://doi.org/10.1111/birt.12316

Toohill, J., Fenwick, J., Gamble, J., Creedy, D. K., Buist, A., Turkstra, E., \& Ryding, E. L. (2014). A randomized controlled trial of a psycho-education intervention by midwives in reducing childbirth fear in pregnant women. Birth (Berkeley, Calif.), 41(4), 384-394. https://doi.org/10.1111/birt.12136

Frohlich, J., \& Kettle, C. (2015). Perineal care. BMJ clinical evidence. 1401. https://pubmed.ncbi.nlm.nih.gov/25752310/

World Health Organization (WHO) (2018). Intrapartum care for a positive childbirth experience. WHO Recommendations. https:/www.who.int/reproductivehealth/publications/intrapartum-care-guidelines/en/

Tang, X., Lu, Z., Hu, D., \& Zhong, X. (2019). Influencing factors for prenatal Stress, anxiety and depression in early pregnancy among women in Chongqing, China. Journal of affective disorders, 253, 292-302. https://doi.org/10.1016/j.jad.2019.05.003 